\title{
Reproducibility of native myocardial T1 mapping in the assessment of Fabry disease and its role in early detection of cardiac involvement by cardiovascular magnetic resonance
}

\author{
Silvia Pica ${ }^{1,2}$, Daniel M Sado ${ }^{1,3}$, Viviana Maestrini ${ }^{1,4}$, Marianna Fontana', Steven K White ${ }^{1,3}$, Thomas Treibel ${ }^{1,3}$, \\ Gabriella Captur $^{1,3}$, Sarah Anderson ${ }^{1}$, Stefan K Piechnik ${ }^{1,5}$, Matthew D Robson ${ }^{1,5}$, Robin H Lachmann ${ }^{6}$, Elaine Murphy ${ }^{6}$, \\ Atul Mehta ${ }^{7}$, Derralyn Hughes ${ }^{7}$, Peter Kellman ${ }^{8}$, Perry M Elliott ${ }^{1,3}$, Anna S Herrey ${ }^{1,3}$ and James C Moon ${ }^{1,3^{*}}$
}

\begin{abstract}
Background: Cardiovascular magnetic resonance (CMR) derived native myocardial $\mathrm{T} 1$ is decreased in patients with Fabry disease even before left ventricular hypertrophy (LVH) occurs and may be the first non-invasive measure of myocyte sphingolipid storage. The relationship of native $\mathrm{T} 1$ lowering prior to hypertrophy and other candidate early phenotype markers are unknown. Furthermore, the reproducibility of $\mathrm{T1}$ mapping has never been assessed in Fabry disease.

Methods: Sixty-three patients, 34 (54\%) female, mean age $48 \pm 15$ years with confirmed (genotyped) Fabry disease underwent CMR, ECG and echocardiographic assessment. LVH was absent in 25 (40\%) patients. Native T1 mapping was performed with both Modified Look-Locker Inversion recovery (MOLLI) sequences and a shortened version (ShMOLLI) at 1.5 Tesla. Twenty-one patients underwent a second scan within 24 hours to assess inter-study reproducibility. Results were compared with 63 healthy age and gender-matched volunteers.
\end{abstract}

Results: Mean native T1 in Fabry disease (LVH positive), (LVH negative) and healthy volunteers was $853 \pm 50 \mathrm{~ms}, 904 \pm$ $46 \mathrm{~ms}$ and $968 \pm 32 \mathrm{~ms}$ (for all $p<0.0001$ ) by ShMOLLI sequences. Native T1 showed high inter-study, intra-observer and inter-observer agreement with intra-class correlation coefficients (ICC) of 0.99, 0.98, 0.97 (ShMOLLI) and 0.98, 0.98, 0.98 (MOLLI). In Fabry disease LVH negative individuals, low native T1 was associated with reduced echocardiographic-based global longitudinal speckle tracking strain $(-18 \pm 2 \%$ vs $-22 \pm 2 \%, p=0.001)$ and early diastolic function impairment $\left(E / E^{\prime}=7[6-8]\right.$ vs $\left.5[5-6], p=0.028\right)$.

Conclusion: Native T1 mapping in Fabry disease is a reproducible technique. T1 reduction prior to the onset of LVH is associated with early diastolic and systolic changes measured by echocardiography.

Keywords: Cardiovascular magnetic resonance, T1 mapping, Speckle-tracking strain, Diastolic function, Fabry disease

\section{Background}

Fabry disease is a rare but treatable X-linked disorder of lysosomal metabolism caused by reduced or absent activity of the alpha galactosidase enzyme, resulting in lysosomal sphingolipid accumulation in a number of different organs. Since the advent of renal replacement therapy, the

\footnotetext{
* Correspondence: j.moon@ucl.ac.uk

'The Heart Hospital, 16-18 Westmoreland Street, London W1G 8PH, UK ${ }^{3}$ Institute of Cardiovascular Science, University College London, London WC1E 6BT, UK

Full list of author information is available at the end of the article
}

principle driver on mortality in Fabry disease is cardiac. Left ventricular hypertrophy (LVH), valve thickening, myocardial scarring, progression to heart failure and sudden arrhythmic death may occur $[1,2]$. Fabry disease patients can be treated with enzyme replacement therapy (ERT) [3]. A number of major challenges in the clinical care of patients with potential Fabry disease remain, such as: (i) the differential diagnosis of Fabry disease-related LVH vs other causes of LVH; (ii) the detection of early cardiac involvement in genetically diagnosed Fabry disease patients (particularly in female heterozygotes); (iii) Timing 
of commencement and monitoring of ERT. The biology of myocardial storage (hypertrophy, fibrosis, cell loss) is not understood, but there is suggestion that therapy should be started early before changes are permanent [4]. However, treatment is sufficiently expensive that early therapy would have societal cost implications [5-7]. New, non-invasive markers of myocardial storage are therefore needed.

Recent advances in cardiovascular magnetic resonance (CMR) allow measurement of native myocardial T1 using a single, short breath-hold mapping sequence [8]. Native $\mathrm{T} 1$ is known to be higher in fibrosis, edema and amyloid, and lower in iron overload and focal fat infiltration [9-11]. In Fabry disease with $\mathrm{LVH}$, both our group and another have shown that native myocardial $\mathrm{T} 1$ is substantially lower compared to other LVH aetiologies [12,13] and around half of Fabry disease patients without LVH have T1 lowering when compared to healthy volunteers [13]. The known lowering of $\mathrm{T} 1$ by fat and the early spectroscopic results suggest that native T1 may be directly measuring myocardial storage in Fabry disease [12]. Native T1 mapping therefore has the potential role in clinical evaluation (prognosis, differential diagnosis and early detection) and management (timing of therapy and follow up) of Fabry disease patients and as a tool to help in understanding the biology of myocardial storage. However, several questions regarding the use of T1 mapping in Fabry disease remain, particularly its reproducibility and whether low native T1 in LVH negative patients is a real marker of early disease with potential functional consequences.

In this study we investigated the reproducibility of native T1 assessment in Fabry disease and the relationship of native T1 in Fabry disease patients with no LVH, looking for functional (electrocardiographic, mechanical) correlations of $\mathrm{T} 1$ reduction.

\section{Methods}

This research received approval from the local research ethics committee and all participants provided written informed consent. Patients were prospectively recruited from the department of inherited cardiovascular disease at The Heart Hospital, London, UK. All patients had a genetically confirmed diagnosis of Fabry disease. This population was compared with gender and age matched cohorts of healthy volunteers: one for the whole population of Fabry disease, one for the male cohort and one for the female cohort, selected from a pool of 63 healthy volunteers, as previously described [13].

CMR was performed on a $1.5 \mathrm{~T}$ magnet (Avanto, Siemens Medical Solutions). Left ventricular volume, mass and ejection fraction were calculated from steady state free precession cine imaging using a thresholding method. $\mathrm{LVH}$ was defined as an elevated indexed left ventricular mass based on body surface area (BSA) normalized cut-off values and stratified for age and gender (Table 1) [14].
Native T1 measurement was performed using a Shortened Modified Look Locker Inversion recovery (ShMOLLI) sequence. In the reproducibility group a second sequence, Modified Look Locker Inversion recovery (MOLLI - sampling scheme $5 \mathrm{~s}(3 \mathrm{~s}) 3 \mathrm{~s}$ with motion correction) was also performed and the patient removed from the scanner, to be re-scanned later in the day; in this group, ShMOLLI and MOLLI T1 assessment were then repeated. The minimum and maximum time between scan 1 and 2 was 8 to 40 minutes. A shim box was placed tightly over the heart as recommended [15]. Optimal gating and breath-holding were ensured and raw images and error maps were examined for potential image artefacts during scanning, to allow an immediate repeat of suboptimal measurements [8]. Following the second T1 assessment, standard late gadolinium enhancement (LGE) imaging was performed on all patients. In patients not undergoing reproducibility, LGE was performed at the end of the first and only scan. Both ShMOLLI and MOLLI T1 sequences were performed using previously published algorithms [16,17]. For ShMOLLI, the resulting pixel-by-pixel color $\mathrm{T} 1$ maps were displayed using a customised 12 bit look up table where normal myocardium was green, increasing T1 red and decreasing T1 blue. The color-map was immediately available after data acquisition. A region of interest (ROI) was drawn directly on each T1 map using ARGUS software (Siemens, Erlangen, Germany) and T1 values averaged between all pixels. T1 was measured in the basal to mid septum in the short axis plane taking care to avoid the blood/myocardial boundary. A second ROI was drawn in basal and mid infero-lateral wall and later compared with LGE images. For test-retest reproducibility study, this process was done for both ShMOLLI and MOLLI sequences.

The analyses were carried out by two blinded observers (SP and DS): SP analyzed the same ShMOLLI and MOLLI mid short axis picture twice for intra-observer reproducibility, DS analysed all studies (including re-test) once, making intra-observer, inter-observer and interstudy data available. For these analyses, all scans were anonymized, i.e. scan 1 and 2 split up.

All patients enrolled underwent ECG and echocardiographic examination within two days of the CMR study. ECG analysis comprised the assessment of cardiac rhythm and axis, PR interval, QRS interval, indices of LVH including RE score [18]. Echocardiography was performed using commercially available systems (Vivid 7 or 9, GE Healthcare). A routine study was performed, according to standard protocols of the European Society of Cardiology, including evaluation of diastolic function with conventional Doppler based measurements (trans-mitral E/A ratio, $\mathrm{E}$ wave deceleration time, pattern of pulmonary vein flow); Tissue Doppler was performed to assess S' and E' mitral annular velocities at the septal and lateral corners, with the measurements being averaged. The E/E' ratio was 
Table 1 BSA-normalized LVmass cut-offs age and gender stratified for LV-hypertrophy definition

\begin{tabular}{lllllll}
\hline & $\mathbf{2 0 - 2 9}$ years & $\mathbf{3 0 - 3 9}$ years & $\mathbf{4 0 - 4 9}$ years & $\mathbf{5 0 - 5 9}$ years & $\mathbf{6 0 - 6 9}$ years & $\mathbf{7 0 - 7 9}$ years \\
\hline Females LVmass/BSA (g/m2) & $62(47-77)$ & $62(47-77)$ & $63(48-77)$ & $63(48-78)$ & $63(48-78)$ & $63(49-78)$ \\
Males LVmass/BSA (g/m2) & $76(59-93)$ & $75(59-92)$ & $75(58-91)$ & $74(57-91)$ & $73(57-90)$ & $73(56-89)$ \\
\hline
\end{tabular}

Adapted from Maceira et. al.

BSA: body surface area; LV: left ventricle.

derived as a measure of LV filling pressures. Digital routine grey scale two-dimensional cine loops from three consecutive beats were obtained from standard apical and short axis views. Systolic 2D strain was calculated by realtime tracking of natural acoustic markers during consecutive frames by 2D strain software as previously described [19]. All data was prospectively and blindly analysed for global and infero-lateral longitudinal, circumferential and radial strain.

Exclusion criteria for this study were usual contraindications to perform a CMR scan. Patients with eGFR $<30 \mathrm{ml} / \mathrm{min}$ were not administered gadolinium contrast and patients with sub-optimal acoustic window or frame rates were excluded from the speckle tracking strain analysis.

\section{Data analysis and statistics}

Data were analysed with JMP ${ }^{\circ}$ 8.0.2 software. Data following a parametric distribution (Shapiro Wilk test $\mathrm{p}>0.05$ ) was described using mean \pm standard deviation. Nonparametric data was described using median and interquartile range. Count and percent were used to describe categorical data. The difference between groups was compared with either the Student $t$ test (parametric) or the Mann Whitney U test (non parametric) and Fisher's exact test for categorical data Correlations between parameters are described either with Pearson $\mathrm{R}$ or with Spearman $\rho$ (rho), depending on their distribution. Inter-study, intraobserver and inter-observer reproducibility of native T1 measurements was assessed by calculating the Intraclass Correlation Coefficient (ICC) with Pearson correlation and Bland Altman plots and the coefficient of variance (COV). A p value $<0.05$ was considered statistically significant.

\section{Results}

\section{Who is in study: baseline characteristics}

Sixty-three patients were included in the study. Of the 63 patients, 44 had previously been studied from a cohort previously published [13]. Two of these were rescanned with reproducibility and 19 new patients were added. The present study added ECG and echocardiographic data for clinical correlations.

Baseline characteristics of Fabry disease and healthy volunteers are showed in Table 2. Seven patients were not administered gadolinium contrast, either due to severe renal impairment (estimated glomerular filtration rate, eGFR, $<30 \mathrm{ml} / \mathrm{min}, \mathrm{n}=5)$, or patient refusal $(\mathrm{n}=2)$. Twelve patients with sub-optimal acoustic window or frame rates were excluded from speckle tracking strain analysis.

\section{Age and gender reference ranges for $\mathrm{T} 1$}

Native T1 is known to be influenced by gender and (to a much lesser extent) age [12,20]. For comparison with healthy controls therefore, on the same scanner/set-up, normal ShMOLLI ranges (and therefore limits of normal) were constructed for Fabry disease male and female cohorts. Age and gender matched comparator groups selected from a pool of 63 healthy volunteer scans were used to identify what the normal and low native $\mathrm{T} 1$ is for male and female population. Using these, the native $\mathrm{T} 1$ ranges were (mean, SD, lower limit of normal): mean $968 \pm 32 \mathrm{~ms}$, lower limit $904 \mathrm{~ms}$ in total population; mean $956 \pm 27 \mathrm{~ms}$, lower limit $902 \mathrm{~ms}$ in male subgroup and mean $978 \pm$ $34 \mathrm{~ms}$, lower limit $910 \mathrm{~ms}$ in female subgroup.

Mean septal T1 in Fabry disease males $(\mathrm{n}=29)$ was lower than in females $(\mathrm{n}=34),(841 \pm 46 \mathrm{~ms}$ vs $901 \pm 45 \mathrm{~ms}$, $\mathrm{p}<0,0001)$.

Table 2 Baseline characteristics of the study population

\begin{tabular}{lcccc}
\hline Parameter & FD global cohort $(\mathbf{n}=\mathbf{6 3})$ & FD LVH negative $(\mathbf{n}=\mathbf{2 5})$ & FD LVH positive $(\mathbf{n}=\mathbf{3 8})$ & Healthy volunteers $(\mathbf{n}=\mathbf{6 3})$ \\
\hline Age (years) & $48 \pm 15$ & $39 \pm 16$ & $54 \pm 11$ & $47 \pm 16$ \\
Sex (M/F) & $29 / 34$ & $6 / 19$ & $23 / 15$ & $29 / 34$ \\
BSA (m2) & $1.8[1.7,2]$ & $1.7[1.6,1.8]$ & $1.9[1.7,2]$ & $1.9[1.7,2]$ \\
LVH (\%) & $38(60)$ & 0 & $38(100)$ & 0 \\
ERT (\%) & $49(78)$ & $14(56)$ & $35(92)$ & 0 \\
Any LGE (\%) & $30(54)$ & $4(17)$ & $26(79)$ & 0 \\
LGE infero-lateral wall & $29(51)$ & $4(17)$ & $25(76)$ & 0 \\
\hline
\end{tabular}

Data are expressed as $n$ (percentage) or mean \pm standard deviation.

BSA: body surface area; LVH: left ventricular hypertrophy; ERT: enzymatic replacing therapy; LGE: late gadolinium enhancement. 
CMR characteristics of Fabry disease individuals divided into two groups (based on the presence or absence of LVH) are shown in Table 3. ECG and Echocardiographic characteristics of Fabry disease subjects are shown in Table 4. The QRS width was directly correlated with left ventricular mass, Pearson $\mathrm{R}=0.72, \mathrm{p}<0.0001$. An example of T1 mapping in Fabry disease individuals is in Figure 1.

\section{T1 in Fabry disease: LVH positive vs LVH negative}

Septal T1 in Fabry disease subjects LVH positive $(\mathrm{n}=38)$ was lower than in LVH negative $(\mathrm{n}=25)$ and healthy volunteers $(n=63),(853 \pm 50 \mathrm{~ms}$ vs $904 \pm 46 \mathrm{~ms}$ vs $968 \pm$ $32 \mathrm{~ms}$ respectively, $\mathrm{p}<0.0001$ ) (Figure 2); there was a weak negative correlation between native T1 and hypertrophy (expressed as left ventricular mass), Pearson $\mathrm{R}=-0.26, \mathrm{p}=0.03$. Of the LVH positive Fabry disease subjects, 34 (89\%) had low T1 (<902 ms for males, $<910 \mathrm{~ms}$ for females). Of the Fabry disease LVH negative subjects, 12 (48\%) had low T1.

\section{T1 in LVH positive population}

Thirty-four of thirty-eight patients (89\%) of LVH positive patient had low septal T1, ranging from $771 \mathrm{~ms}$ to 908 ms. The 4 Fabry disease subjects with 'normal' septal T1 were females, 3 of them with mild LVH or apical prevalent $\mathrm{LVH}$ and 2 of them with basal infero-lateral LGE and $>3$ years of ERT (minimum 5, maximum 12 years). One subject, 44 years, had severe concentric hypertrophy, T1 = $978 \mathrm{~ms}, 2$ years of ERT and extensive
LGE. For this small group $(\mathrm{n}=4)$, standard and advanced echo and CMR parameters were the same as the low T1 LVH positive group. The ECG (QRS) was narrower in this group however $(94 \pm 7 \mathrm{~ms}$ vs $117 \pm 23 \mathrm{~ms}$, $\mathrm{p}<0.01)$.

Regionally, native T1 in the infero-lateral wall tracked the presence $(n=25)$ or absence $(n=9)$ of LGE in this area (LGE+ native T1 $919 \pm 63 \mathrm{~ms}$ vs LGE- native T1 $848 \pm 32 \mathrm{~ms}, \mathrm{p}<0.01)$

\section{T1 in LVH negative population}

Of the LVH negative subjects who were administered contrast ( $n=23), 4(17 \%)$ had LGE, always in the inferolateral wall. This was associated with normal mean $\mathrm{T} 1$ in the infero-lateral segment as compared with lower T1 in subjects without LGE $(954 \pm 15 \mathrm{~ms}$ vs $888 \pm 41 \mathrm{~ms}$, $\mathrm{p}<0.0001)$. We found no correlation between mean septal T1 and LGE. Mean septal T1 was comparable in patients with and without LGE $(902 \pm 49$ ms vs $908 \pm 46$ ms, p 0.8$)$

Fabry disease patients without LVH had a mean septal $\mathrm{T} 1$ at the lower limit of normal range (904 $\pm 46 \mathrm{~ms})$, ranging from $818 \mathrm{~ms}$ to $979 \mathrm{~ms}$ (Figure 2).

Based on the ShMOLLI mean value of $956 \pm 27 \mathrm{~ms}$ for males and $978 \pm 34 \mathrm{~ms}$ for females, we divided our population into four groups, according to presence/absence of $\mathrm{LVH}$ and low/normal native septal T1, (-2SD below healthy mean: $902 \mathrm{~ms}$ cut-point for males and $910 \mathrm{~ms}$ cutpoint for females). Group 1: LVH negative-T1N, $\mathrm{n}=13$;

Table 3 CMR characteristics of Fabry disease patients divided into LVH positive/LVH negative and Healthy volunteers

\begin{tabular}{|c|c|c|c|c|}
\hline & Healthy volunteers $(n=63)$ & FD LVH negative $(n=25)$ & FD LVH positive $(n=38)$ & p-value ${ }^{t}$ \\
\hline LV Mass i (g/m2) & $66 \pm 14$ & $72 \pm 13$ & $132 \pm 46$ & $<0.01$ \\
\hline Max wall thickness (mm) & & $8 \pm 2$ & $16 \pm 4$ & $<0.01$ \\
\hline LVEDVi (ml/m2) & $72 \pm 13$ & $70 \pm 15$ & $67 \pm 16$ & 0.30 \\
\hline LVESVi (ml/m2) & $24 \pm 7$ & $17 \pm 6$ & $16 \pm 6$ & 0.10 \\
\hline LVEF (\%) & $67 \pm 5$ & $74 \pm 6$ & $78 \pm 7$ & 0.05 \\
\hline Left atrial area i (cm2/m2) & $11 \pm 2$ & $12 \pm 3$ & $13 \pm 3$ & 0.03 \\
\hline LGE & 0 & $4(17)$ & $26(79)$ & $<0.01$ \\
\hline infero-lateral wall & & $4(17)$ & $25(76)$ & $<0.01$ \\
\hline extensive & & 0 & $2(6)$ & \\
\hline RV inserction points & & 0 & 1(3) & \\
\hline Average septal T1 (ms)* & $968 \pm 32$ & $904 \pm 46$ & $853 \pm 50$ & $<0.01$ \\
\hline Average infero-lateral T1 (ms)* & & $894 \pm 65$ & $903 \pm 45$ & 0.27 \\
\hline \multicolumn{5}{|l|}{ Average infero-lateral T1 (ms)* } \\
\hline LGE yes & & $954 \pm 15$ & $919 \pm 63$ & 0.01 \\
\hline LGE no & & $888 \pm 41$ & $848 \pm 32$ & $<0.01$ \\
\hline
\end{tabular}

Data are expressed as $n$ (percentage) or mean \pm standard deviation. *native T1 measured by ShMOLLI sequences.

${ }^{+} \mathrm{p}$ value between $\mathrm{LVH}$ negative and LVH positive groups. LVEDV: left ventricular end-diastolic volume; LVESV: left ventricular end-systolic volume; LGE: late gadolinium enhancement; LVEF: left ventricular ejection fraction, $\mathrm{RV}$ : right ventricle. 
Table 4 ECG and Echocardiographic characteristics of Fabry disease patients divided into LVH negative and LVH positive

\begin{tabular}{|c|c|c|c|}
\hline & LVH negative $(n=25)$ & LVH positive $(n=38)$ & $\mathrm{p}$-value \\
\hline PR interval (ms) & $149 \pm 32$ & $158 \pm 29$ & 0.25 \\
\hline QRS interval (ms) & $92[85,100]$ & $108[96,130]$ & $<0.01$ \\
\hline Axis (degree) & $60[47,74]$ & $38[-12,63]$ & $<0.01$ \\
\hline TWI & $3(12)$ & $30(79)$ & $<0.01$ \\
\hline Sokolow (Sv1 + RV5/6) (mm) & $32 \pm 9$ & $37 \pm 18$ & 0.06 \\
\hline RE score $\geq 4$ & $3(12)$ & $32(84)$ & $<0.01$ \\
\hline$E / E^{\prime}$ & $6[5,8]$ & $10[8,13]$ & $<0.01$ \\
\hline Systolic velocity Doppler TDI (m/s) & $0.09 \pm 0.02$ & $0.06 \pm 0.01$ & $<0.01$ \\
\hline Left atrial volume (ml) & $60 \pm 18$ & $91 \pm 28$ & $<0.01$ \\
\hline Global longitudinal strain (\%) & $-20 \pm 3$ & $-13 \pm 4$ & $<0.01$ \\
\hline Infero-lateral longitudinal strain (\%) & $-21 \pm 3$ & $-13 \pm 4$ & $<0.01$ \\
\hline Global basal radial strain (\%) & $38 \pm 19$ & $34 \pm 12$ & 0.22 \\
\hline Infero-lateral basal radial strain (\%) & $44 \pm 14$ & $34 \pm 15$ & 0.02 \\
\hline Global basal circumferential strain (\%) & $-18 \pm 4$ & $-15 \pm 5$ & 0.03 \\
\hline Infero-lateral basal circumf strain (\%) & $-13 \pm 5$ & $-10 \pm 8$ & 0.05 \\
\hline TAPSE (mm) & $23 \pm 4$ & $20 \pm 3$ & $<0.01$ \\
\hline
\end{tabular}

Data are expressed as $\mathrm{n}$ (percentage) or mean \pm standard deviation or median [interquartile range].

TWI: T wave inversion; TDI: Tissue Doppler Imaging; TAPSE: tricuspid annular plane systolic excursion.

Group 2: LVH negative-T1 $\downarrow, \mathrm{n}=12$; Group 3: LVH positive-T1 $\downarrow, \mathrm{n}=34$; Group 4: LVH positive-T1N, $\mathrm{n}=4$.

\section{Significance of T1 lowering in LVH negative Fabry disease subjects.}

There were 25 Fabry disease patients without LVH: 13 with normal $\mathrm{T} 1$ and 12 with low T1. Comparing these groups, the ECG was the same between groups. Standard CMR and Echo parameters for heart size and function were the same, but advanced echocardiography techniques revealed differences in systolic and diastolic function with slightly lower global longitudinal speckle tracking strain, and reduced diastolic function based on E/ E' in LVH negative-T1 $\downarrow$ subjects (Figure 5). This also

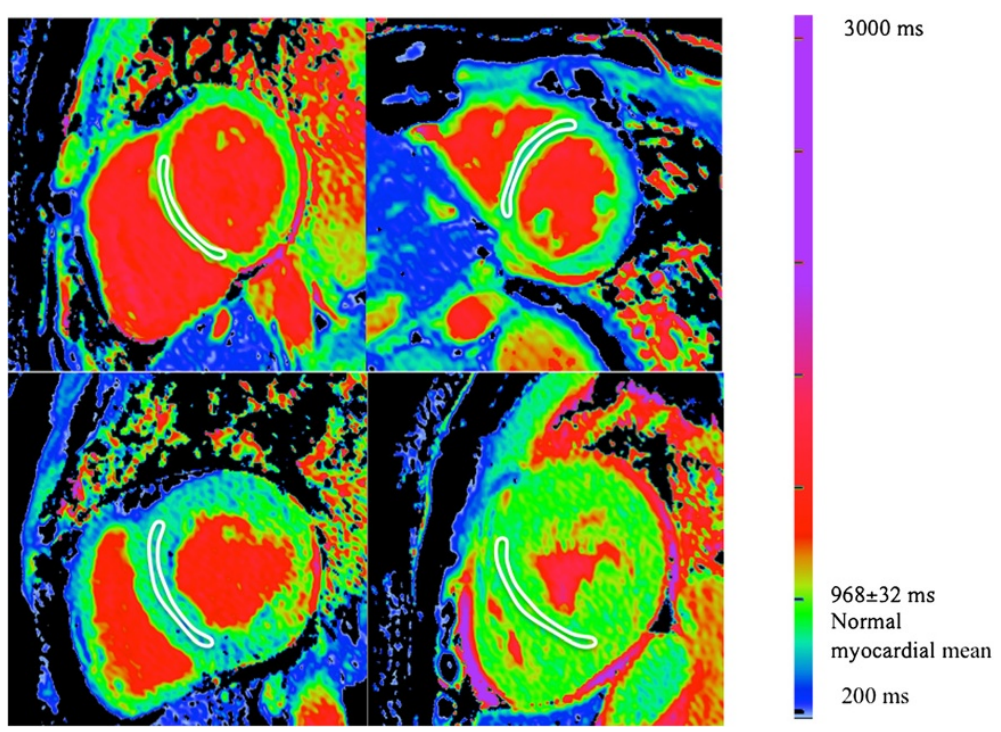

Figure 1 Native T1 mapping in Fabry disease using ShMOLLI at 1.5 T. Top left: normal. Top right: a Fabry disease subject without LVH but clear myocardial T1 reduction - the myocardium is blue. Bottom left: Typical T1 when LVH present: the myocardial T1 is lower than without LVH and the basal infero-lateral wall has T1 elevation with a normal (pseudonormal?) surrounding area. Bottom right: rarely (4 patients), Fabry disease has a normal $\mathrm{T}$. 


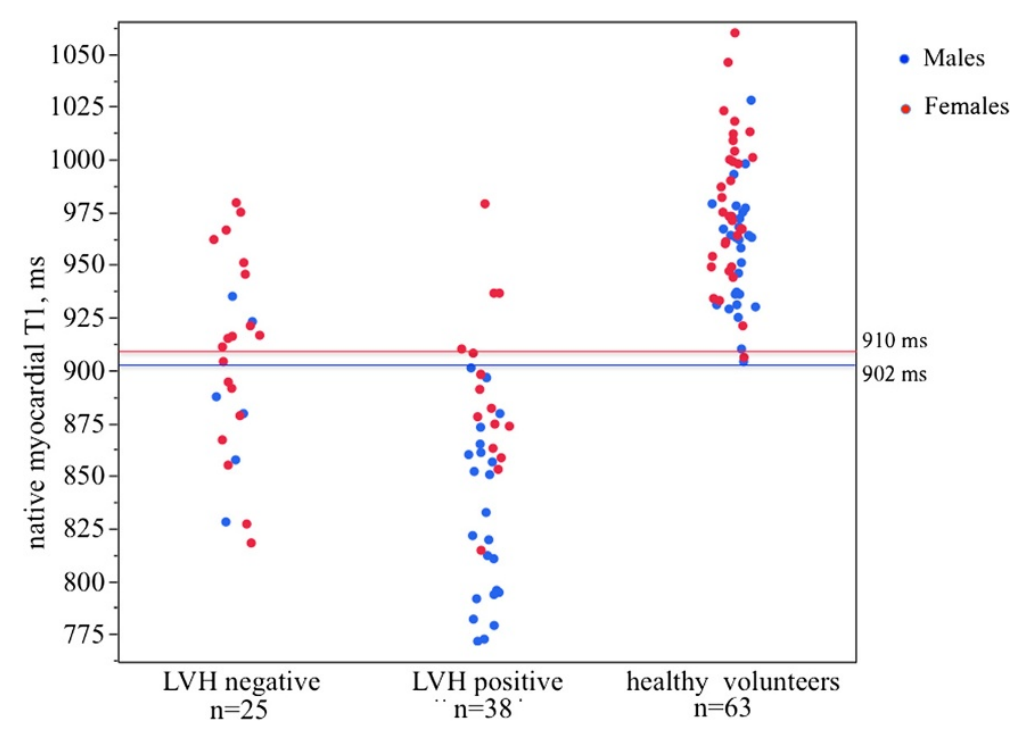

Figure 2 Mean septal T1 in LVH negative patients $(n=25)$, LVH positive patients $(n=38)$ and healthy volunteers $(n=63)$. Red solid line indicates -2SD below the mean native T1 of females healthy volunteers. Blue solid line indicates -2SD below the mean native T1 of males healthy volunteers.

tracked the degree of T1 lowering (T1 vs strain Pearson R -0.41 , p 0.07; T1 vs E/E' Spearman rho $=-0.51, \mathrm{p}=0.01$ ), Table 5 and Figure 3. Supporting diastolic impairment, left atrial size was bigger in LVH negative-T1 $\downarrow$ subjects than in LVH negative-T1N.

Considering the cut off value of $902 \mathrm{~ms}$ for males and $910 \mathrm{~ms}$ for females, T1 had 48\% sensitivity (i.e. half did not have low T1), but 99\% specificity in distinguishing between Fabry disease LVH negative subjects and healthy volunteers (Receiver operating characteristic AUC 0.73).

ShMOLLI and MOLLI sequences derived exactly the same results but with, as previously described, a sequence design related $60 \mathrm{~ms}$ offset (MOLLI higher) [21].

Table 5 Echocardiographic, CMR, ECG characteristics of LVH negative individuals with normal (LVH negative-T1N) or low T1 (LVH negative-T1 $\downarrow$ )

\begin{tabular}{|c|c|c|c|}
\hline & LVH negative-T1N, $(n=13)$ & LVH negative-T1 $\downarrow,(n=12)$ & p-value \\
\hline$E / E^{\prime}$ & $5[5,6]$ & $7[6,8]$ & 0.03 \\
\hline Global longitudinal strain (\%) & $-22 \pm 2$ & $-18 \pm 2$ & $<0.01$ \\
\hline Infero-lateral longitudinal strain (\%) & $-23 \pm 3$ & $-20 \pm 4$ & 0.01 \\
\hline Global basal radial strain (\%) & $37 \pm 24$ & $39 \pm 13$ & 0.36 \\
\hline Infero-lateral basal radial strain (\%) & $45 \pm 15$ & $42 \pm 15$ & 0.70 \\
\hline Global basal circumferential strain (\%) & $-18 \pm 5$ & $-18 \pm 4$ & 0.99 \\
\hline Infero-lateral basal circumf strain (\%) & $-15 \pm 6$ & $-12 \pm 3$ & 0.12 \\
\hline TAPSE (mm) & $23 \pm 4$ & $23 \pm 4$ & 0.96 \\
\hline Systolic velocity Doppler TDI (m/s) & $0.09 \pm 0.02$ & $0.08 \pm 0.02$ & 0.36 \\
\hline PR interval (ms) & $148 \pm 22$ & $150 \pm 42$ & 0.45 \\
\hline QRS interval (ms) & $93 \pm 9$ & $95 \pm 11$ & 0.26 \\
\hline TWI & $1(8)$ & $2(17)$ & 0.56 \\
\hline RE score $\geq 4$ & $2(15)$ & $1(8)$ & 0.46 \\
\hline Left atrial area $\mathrm{i}(\mathrm{cm} 2 / \mathrm{m} 2)^{*}$ & $11 \pm 2$ & $13 \pm 2$ & $<0.01$ \\
\hline LVEDVi $(\mathrm{ml} / \mathrm{m} 2)^{*}$ & $67 \pm 7$ & $72 \pm 19$ & 0.22 \\
\hline LV mass i $(\mathrm{g} / \mathrm{m} 2)^{*}$ & $69 \pm 10$ & $76 \pm 16$ & 0.19 \\
\hline
\end{tabular}

Data are expressed as $\mathrm{n}$ (percentage) or mean \pm standard deviation or median [interquartile range].

TDI: Tissue Doppler Imaging; TAPSE: tricuspid annular plane systolic excursion; TWI: T wave inversion; LVEDV: left ventricular end-diastolic volume;

*by CMR. 

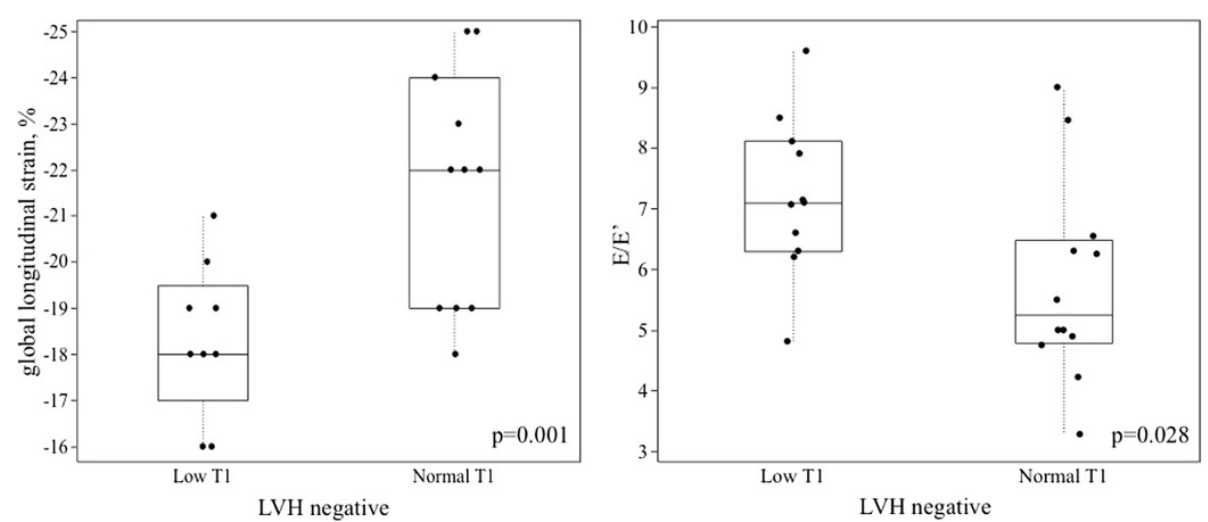

Figure 3 Systolic and diastolic function in LVH negative Fabry disease patients. Global longitudinal speckle tracking strain of LVH negativeT1 $\downarrow$ subjects vs LVH negative-T1N subjects (left); E/E' of LVH negative-T1 $\downarrow$ subjects vs LVH negative-T1N subjects (right).

\section{Reproducibilty}

T1 mapping was highly reproducible regardless of methodology used (Table 6): inter-study reproducibility for ShMOLLI sequences showed an intraclass correlation coefficients (ICC) of 0.992 and relative difference between the means of $-0.3 \%$ ( $95 \% \mathrm{CI}-4.6 \%$ to $4.0 \%$ ) (Figure 4 ). Intra-observer and inter-observer reproducibility were similarly very good with relative difference between the means of $-0.3 \%$ ( $95 \%$ CI $-4.6 \%$ to $4.0 \%$ ) and $-0.8 \%$ ( $95 \%$
CI $-3.5 \%$ to $5.0 \%)$ respectively. ShMOLLI and MOLLI sequences showed the same reproducibility.

\section{Discussion}

As previously described, decreased native myocardial T1 is highly prevalent in Fabry disease patients with LVH. We show in addition that in subjects without LVH, reduced myocardial $\mathrm{T} 1$ has a $50 \%$ prevalence and is associated with echocardiographic parameters of cardiac
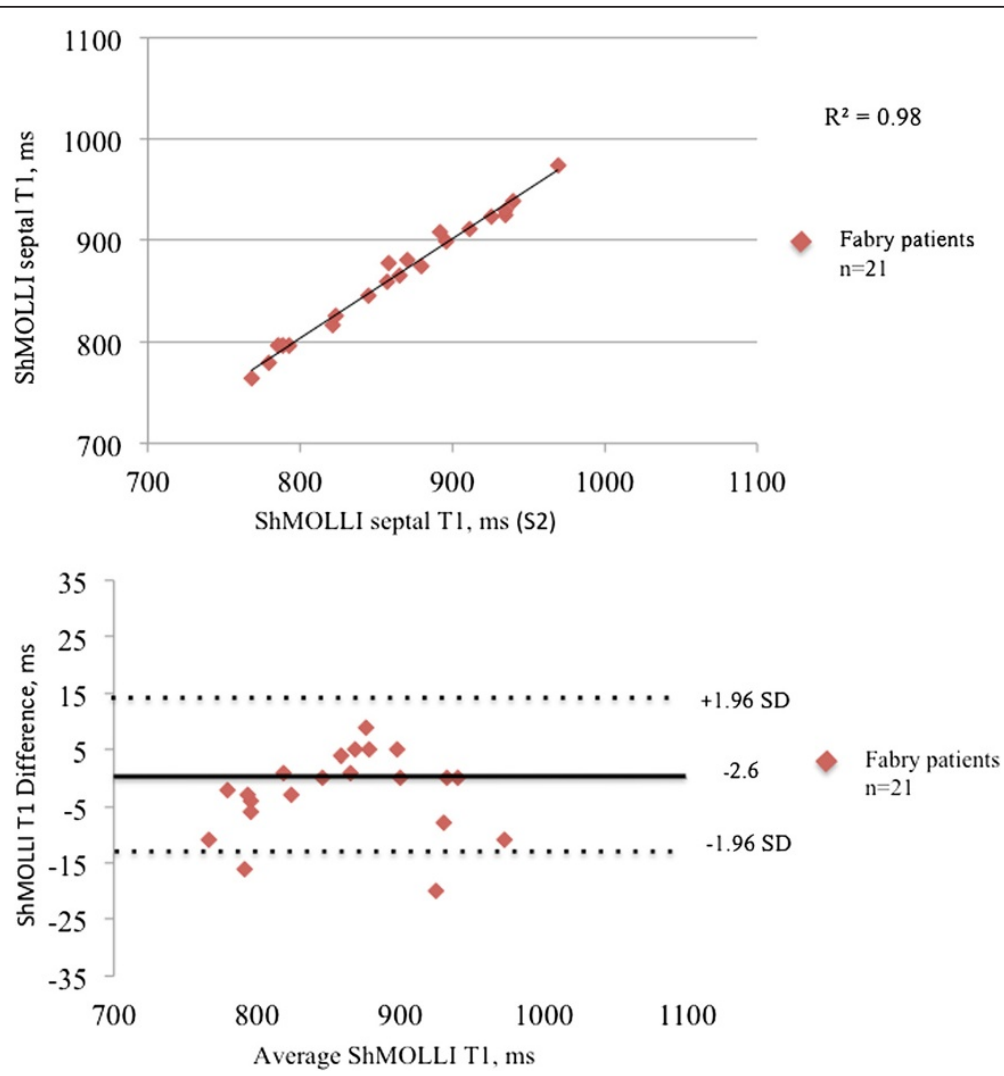

Figure 4 Reproducibility of ShMOLLI sequences. ShMOLLI inter-study correlation (upper pannel) and Bland Altman analysis (lower pannel). 
Table 6 ShMOLLI and MOLLI T1 reproducibility

\begin{tabular}{llll}
\hline & Inter-study ICC (COV\%) & Intra-observer ICC (COV\%) & Inter-observer ICC (COV\%) \\
\hline ShMOLLI T1 & $0.992(0.8 \%)$ & $0.976(1.5 \%)$ & $0.973(1.4 \%)$ \\
MOLLI T1 & $0.978(1.5 \%)$ & $0.978(1.2 \%)$ & $0.982(1.1 \%)$ \\
\hline
\end{tabular}

ICC: intraclass correlation coefficient; COV: coefficient of variance.

dysfunction suggesting that a low T1 is detecting early cardiac disease. As a candidate biomarker, native myocardial T1 in Fabry disease is useful both in established and early disease, and with both MOLLI and ShMOLLI approaches.

There are many current clinical challenges in Fabry disease. For example, the diagnosis is often missed and there is uncertainty as when to begin ERT and also how to monitor its effects. CMR derived myocardial T1 mapping has previously been shown to have very high sensitivity and specificity to discriminate Fabry disease patients with LVH $[12,13]$. Low T1 in Fabry disease is likely a consequence of the progressive sphingolipid storage in the myocardium. We suspect that it may be the specific storage pattern of lipid in Fabry disease i.e. the lamellar bodies with water restricted between layers, reminiscent of the myelin sheath rather than just the presence of fat [13].

We have previously proposed 4 phases of myocardial Fabry disease (with these data adding myocardial dysfunction to phase 2): phase 1: normal; phase 2: low T1, early myocardial dysfunction; phase $3, \mathrm{LVH}$ low T1 and phase 4: 'pseudonormalisation' of $\mathrm{T} 1$, fibrosis and heart failure. This study shows that low T1 is not quite ubiquitous in $\mathrm{LVH}$ positive patients with 4 patients not having a reduced septal T1. Whilst one of these may have been in a progressive disease phase 4 , where scarring is causing "pseudonormalisation" of $\mathrm{T} 1$, the other 3 were female heterozygotes with early disease or with an apical HCM like LVH pattern and were on ERT. One tantalising explanation is that ERT in early disease may normalise T1. The efficacy of ERT appears to decrease with the progression of the disease and may be unsuccessful when marked fibrosis has been established $[6,22]$. If storage can be removed before the myocyte and myofibroblast activate and induce hypertrophy and fibrosis, cardiac disease could be avoided. The link of low T1 to early dysfunction may indicate the early stages of such activation. These $50 \%$ of $\mathrm{LVH}$ negative individuals with a low T1 could therefore be a target for early ERT. The reproducibility data for native $\mathrm{T} 1$ has been documented in other diseases $[10,20,23,24]$ and here appears to be a reliable marker in Fabry disease, at least when performed on the same magnet. There are ongoing research programs working on standardization between magnets.

This reproducibility is not found in advanced echocardiographic parameters where increased sensitivity between groups (here lower global longitudinal strain and a worse E/E' ratio) has not yet been matched with robust inter-subject reproducibility and the ability to guide therapy in an individual [19,25-28]. This provides three potential advantages: first, a potentially clear risk stratification and therapy targeting method; secondly, a way of clinically monitoring therapy; and thirdly, a surrogate endpoint for drug development, dose ranging and clinical trials. An integrated imaging approach including both cardiac magnetic resonance and echocardiographic parameters might be optimal for the management of these patients.

\section{Limitations}

CMR, ECG and echocardiographic findings were not compared to the gold standard of cardiac biopsy, or candidate measures of myocardial storage such as CMR derived spectroscopy. The reproducibility work is single centre, single magnet and therefore further work will be required to assess these variables in scanners from different vendors and at 3 Tesla field strength. A potential limitation of the family of T1 mapping methods used in this paper is the dependence of the $\mathrm{T} 1$ values on other physical parameters such as T2 and magnetization transfer (MT), which have been shown to affect apparent $\mathrm{T} 1$ values [29].

Furthermore, because of small numbers especially in LVH negative group, separate analysis by gender or prior ERT use were prevented. Future work will need to be larger, multicentre and look at both disease progression and impact of therapy. Lastly, we didn't measure extracellular volume (ECV) in our population. Because ECV is a ratio, ECV might be more comparable across platforms and sequences. However, we didn't measure ECV based on previous data from our center, showing ECV didn't raise in AFD patients.

\section{Conclusions}

Native T1 mapping is highly reproducible in Fabry disease using either ShMOLLI or MOLLI sequences. In Fabry disease LVH negative individuals, low native T1 tracks early myocardial dysfunction and appears to be a promising tool to detect early cardiac involvement in Fabry disease individuals.

\section{Competing interests}

The authors declare that they have no competing interests.

\section{Authors' contributions}

SP: led the study; co-ordinated analysis; lead writer of manuscript. SKP: technical support for T1 mapping sequence/ShMOLLI development, analysis of T1 maps. DS: blind analysis of ShMOLLI and MOLLI data, healthy volunteer and original Fabry disease cohort lead. JCM: concept and design of study. All authors read and approved the final manuscript. 


\section{Acknowledgments}

Authors are grateful to all the staff of the Heart Hospital Imaging Centre and the Cardiomyopathies Clinic for the support received which made this manuscript possible.

\section{Author details \\ ${ }^{1}$ The Heart Hospital, 16-18 Westmoreland Street, London W1G 8PH, UK. ${ }^{2}$ Department of Cardiology, IRCCS Policlinico San Matteo Hospital, Pavia, Italy. ${ }^{3}$ Institute of Cardiovascular Science, University College London, London WC1E 6BT, UK. ${ }^{4}$ Department of Cardiovascular, Respiratory, Nephrologic, Anaesthesiologic and Geriatric Science, La Sapienza University of Rome, Rome, Italy. ${ }^{5}$ Oxford Centre for Clinical Magnetic Resonance Research, Department of Cardiovascular Medicine, University of Oxford, Oxford OX3 9DU, United Kingdom. ${ }^{6}$ The National Hospital for Neurology and Neurosurgery, Queens Square, London, UK. 'ysosomal Storage Disorders Unit, Royal Free Hospital and University College London, London, UK. ${ }^{8}$ National Heart, Lung, and Blood Institute, National Institutes of Health, Bethesda, MD, USA.}

Received: 30 June 2014 Accepted: 17 November 2014

Published online: 05 December 2014

\section{References}

1. O'Mahony C, Elliott P. Anderson-fabry disease and the heart. ProgCardiovasc Dis. 2010; 52(4):326-35.

2. Mehta A, Clarke JTR, Giugliani R, Elliott P, Linhart A, Beck M, Sunder-Plassmann $G$. Natural course of fabry disease: changing pattern of causes of death in FOS - fabry outcome survey. J Med Genet. 2009; 46(8):548-52.

3. Hughes DA, Elliott PM, Shah J, Zuckerman J, Coghlan G, Brookes J, Mehta $A B$. Effects of enzyme replacement therapy on the cardiomyopathy of anderson-fabry disease: a randomised, double-blind, placebo-controlled clinical trial of agalsidase alfa. Heart. 2008; 94(2):153-58.

4. Moon JC, Sachdev B, Elkington AG, McKenna WJ, Mehta A, Pennell DJ, Leed PJ, Elliott PM. Gadolinium enhanced cardiovascular magnetic resonance in anderson-fabry disease. Evidence for a disease specific abnormality of the myocardial interstitium. Eur Heart J. 2003; 24:2151-55.

5. Deegan PB, Baehner AF, Barba Romero MA, Hughes DA, Kampmann C, Beck M. Natural history of fabry disease in females in the fabry outcome survey. J Med Genet. 2006; 43(4):347-52.

6. Taciane A, Filippo V, V De Souza M, C Krug B, V D Schwartz I. Enzyme replacement therapy for fabry disease: a systematic review and meta-analysis. Genet Mol Biol. 2012; 35(4(suppl)):947-54.

7. Banikazemi M, Bultas J, Waldek S, Wilcox WR, Whitley CB, McDonald M, Finkel R, Packman S, Bichet DG, Warnock DG, Desnick RJ, Fabry Disease Clinical Trial Study Group. Agalsidase-beta therapy for advanced fabry disease: a randomized trial. Ann Intern Med. 2007; 146:77-86.

8. Moon JC, Messroghli DR, Kellman P, Piechnik SK, Robson MD, Ugander M, Gatehouse PD, Arai AE, Friedrich MG, Neubauer S, Schulz-Menger J, Schelbert EB. Myocardial T1 mapping and extracellular volume quantification: a Society for Cardiovascular Magnetic Resonance (SCMR) and CMR Working Group of the European Society of Cardiology consensus statement. J Cardiovasc Magn Reson. 2013; 15:92.

9. Dass S, Suttie JJ, Piechnik SK, Ferreira VM, Holloway CJ, Banerjee R, Mahmod M, Cochlin L, Karamitsos TD, Robson MD, Watkins H, Neubauer S. Myocardial tissue characterization using magnetic resonance noncontrast T1 mapping in hypertrophic and dilated cardiomyopathy. Circ Cardiovasc Imaging. 2012; 5(6):726-33.

10. Sado DM, Maestrini V, Piechnik SK, Banypersad SM, White SK, Flett AS, Robson MD, Neubauer S, Ariti C, Arai A, Kellman P, Yamamura J, Schoennagel BP, Shah F, Davis B, Trompeter S, Walker M, Porter J, Moon JC. Noncontrast myocardial T1 mapping using cardiovascular magnetic resonance for iron overload. J Magn Reson Imaging. 2014; doi: 10.1002/ jmri.24727.

11. Ferreira VM, Piechnik SK, Dall'Armellina E, Karamitsos TD, Francis JM, Ntusi N, Holloway C, Choudhury RP, Kardos A, Robson MD, Friedrich MG, Neubauer S. $T(1)$ mapping for the diagnosis of acute myocarditis using CMR: comparison to T2-weighted and late gadolinium enhanced imaging. JACC Cardiovasc Imaging. 2013; 6(10):1048-58.

12. Thompson RB, Chow K, Khan A, Chan A, Shanks M, Paterson I, Oudit GY. $\mathrm{T}_{1}$ mapping with cardiovascular MRI is highly sensitive for fabry disease independent of hypertrophy and sex. Circ Cardiovasc Imaging. 2013; 6:637-45.
13. Sado DM, White SK, Piechnik SK, Banypersad SM, Treibel T, Captur G, Fontana M, Maestrini V, Flett AS, Robson MD, Lachmann RH, Murphy E, Mehta A, Hughes D, Neubauer S, Elliott PM, Moon JC. Identification and assessment of anderson fabry disease by cardiovascular magnetic resonance non-contrast myocardial T1 mapping. Circ Cardiovascular Imaging. 2013; 6(3):392-98.

14. Maceira AM, Prasad SK, Khan M, Pennell DJ. Normalized left ventricular systolic and diastolic function by steady state free precession cardiovascular magnetic resonance. J Cardiovasc Magn Reson. 2006; 8(3):417-26.

15. Kellman P, Herzka DA, Arai AE, Michael Schacht H. Influence of Off-resonance in myocardial T1-mapping using SSFP based MOLLI method. J CardiovasC Magn Reson. 2013; 15:63.

16. Lee JJ, Liu S, Nacif MS, Ugander M, Han J, Kawel N, Sibley CT, Kellman P, Arai $A E$, Bluemke DA. Myocardial $\mathrm{T} 1$ and extracellular volume fraction mapping at 3 Tesla. J Cardiovasc Magn Reson. 2011; 28:13-75.

17. Messroghli DR, Radjenovic A, Kozerke S, Higgins DM, Sivananthan MU, Ridgway JP. Modified Look-Locker inversion recovery (MOLLI) for highresolution T1 mapping of the heart. Magn Reson Med. 2004; 52:141-46.

18. Romhilt DW, Bove KE, Norris RJ, Conyers E, Conradi S, Rowlands DT, Scott RC. A critical appraisal of the electrocardiographic criteria for the diagnosis of left ventricular hypertrophy. Circulation. 1969; 40:185-96.

19. Leitman M, Lysyansky P, Sidenko S, Shir V, Peleg E, Binenbaum M, Kaluski E, Krakover $R$, Vered $Z$. Two-dimensional strain-a novel software for real-time quantitative echocardiographic assessment of myocardial function. J Am SocEchocardiogr. 2004; 10:1021-29.

20. Piechnik SK, Ferreira VM, Lewandowski AJ, Ntusi NA, Banerjee R, Holloway C, Hofman MB, Sado DM, Maestrini V, White SK, Merzaka L, Theodoros K, Moon JC, Stefan N, Paul L, Robson MD. Normal variation of magnetic resonance T1 relaxation times in the human population at 1.5 T using ShMOLLI. J Cardiovasc Magn Reson. 2013; 15(1):13.

21. Kellman P, S Hansen M. T1-mapping in the heart: accuracy and precision. J Cardiovasc Magn Reson. 2014; 4(16(1)):2.

22. Weidemann F, Niemann M, Breunig F, Hermann S, Beer M, Stork S, Voelker W, Ertl G, Ertl G, Wanner C, Strotmann J. Long-term effects of enzyme replacement therapy on fabry cardiomyopathy: evidence for a better outcome with early treatment. Circulation. 2009; 119(4):524-29.

23. Fontana M, White SK, Banypersad SM, Sado DM, Viviana M, Flett AS, Piechnik SK, Stefan N, Neil R, Moon JC. Comparison of T1 mapping techniques for ECV quantification. Histological validation and reproducibility of ShMOLLI versus multibreath-hold T1 quantification equilibrium contrast CMR. J Cardiovasc Magn Reson. 2012; 14:88.

24. Roujol S, Weingartner S, Foppa M, Chow K, Kawaji K, Ngo LH, Kellman P, Manning WJ, Thompson RB, Nezafat R. Accuracy, precision, and reproducibility of four T1 mapping sequences: a head-to-head comparison of MOLLI, ShMOLLI, SASHA, and SAPPHIRE. Radiology. 2014; 4:140296. Epub ahead of print

25. Weidemann F, Breunig F, Beer M, Sandstede J, Turschner O, Voelker W, Ert G, Knoll A, Wanner C, Strotmann JM. Improvement of cardiac function during enzyme replacement therapy in patients with Fabry disease: a prospective strain-rate imaging study. Circulation. 2003; 108:1299-301.

26. Serri $K$, Reant $P$, Lafitte $M$, Berhouet $M$, Le Bouffos V, Roudaut R, Lafitte $S$. Global and regional myocardial function quantification by Twodimensional strain: application in hypertrophic cardiomyopathy. J Am Coll Cardiol. 2006; 47(6):1175-81.

27. Fontana A, Zambon A, Cesana F, Giannattasio C, Trocino G. Tissue doppler, Triplane echocardiography, and speckle tracking echocardiography: different ways of measuring longitudinal myocardial velocity and deformation parameters. A comparative clinical study. Echocardiography. 2012; 29:428-37.

28. Shanks M, B Thompson R, D Paterson I, Putko B, Khan A, Chan A, Becher H, Oudit GY. Systolic and diastolic function assessment in fabry disease patients using speckle-tracking imaging and comparison with conventional echocardiographic measurements. J Am Soc Echocardiography. 2013; 26:1407-14.

29. Robson MD, Piechnik SK, Tunnicliffe EM, Neubauer S. T $T_{1}$ measurements in the human myocardium: the effects of magnetization transfer on the SASHA and MOLLI sequences. Magn Reson Med. 2013; 70:664-70.

\section{doi:10.1186/s12968-014-0099-4}

Cite this article as: Pica et al:: Reproducibility of native myocardial T1 mapping in the assessment of Fabry disease and its role in early detection of cardiac involvement by cardiovascular magnetic resonance. Journal of Cardiovascular Magnetic Resonance 2014 16:99. 